\title{
DESIGN AND SIMULATION OF CURRENT FEEDBACK OPERATIONAL AMPLIFIER IN 180nm AND 90nm CMOS PROCESSES
}

\author{
Sajin C.S. ${ }^{1}$, Ramrosh M.R. ${ }^{2}$ and Shahul Hameed T.A. ${ }^{3}$ \\ ${ }^{I}$ Department of Electronics and Communication Engineering, LBS Centre for Science and Technology, India \\ ${ }^{2,3}$ Department of Electronics and Communication Engineering, T.K.M. College of Engineering, India
}

\begin{abstract}
This paper proposes a Current Feedback Operational Transconductance Amplifier (CFB OTA) with a folded cascode op-amp as input stage cascaded with class $A B$ buffer as output stage. This gives better results on parameters such as bandwidth, slew rate, settling time and input offset voltage compared to the conventional CFOA. Hence it carries significance in high frequency applications such as $A / D$ converters and active filters where $\mathrm{CMOS} C \mathrm{CFOA}$ used as an active building block. CADENCE simulation results are obtained using $180 \mathrm{~nm}$ CMOS process (supply voltage of $\pm 2.5 \mathrm{~V}, 100 \mu \mathrm{A}$ biasing current) and 90nm CMOS process (supply voltage of $\pm 1.2 \mathrm{~V}, 50 \mu \mathrm{A}$ biasing current). Some of the performance parameters obtained are $D C$ gain of 60.2dB, open-loop gain bandwidth product $(\mathrm{GBW})$ of $307 \mathrm{MHz}$, slew rate $(S R+)$ of $+30 V / \mu S$ to $+90 V / \mu S$ and $D C$ input offset voltage of $-0.6 n V$.
\end{abstract}

Keywords:

CFOA, Class AB Buffer, Extended Bandwidth

\section{INTRODUCTION}

Design of analog circuit plays a pivotal role [1] in mixed signal applications, since interfacing digital systems to the analog counterpart in real time applications poses many tangible problems in terms of gain, bandwidth and noise figure. This is true in major application areas like industrial processes, signal conditioning, telecommunication and biomedical measurements. In analog systems, design and implementing analog filters carry significance, particularly in signal processing applications. In analog filters, which are active filters where operational amplifiers form the active element, design criteria are too critical for varying requirements and linearity plays a pivotal role in design [2]. The high gain amplifiers, as operational amplifiers are commonly attributed, it offers good bandwidth at the cost of temperature performance, power dissipation etc. A topology employing CFA-OTA as input stage cascaded with class AB cross coupled buffer as output stage in $.35 \mu \mathrm{m}$ is discussed in paper [1]. A Ka band linear power amplifier is implemented in $28 \mathrm{~nm}$ bulk technology in paper [2]. Also another CMOS CFOA design with $100 \mathrm{MHz}$ bandwidth is presented in paper [11]. The paper given in [14] discusses the theoretical background of the current feedback operational transconductance amplifier

In high frequency and high speed applications, current mode op-amps are frequently used for better performance. One such opamp is Current Feedback Operational trans-conductance Amplifier (CFOA) which is a four terminal device. The main advantage of the CFOA is that it can be easily designed from current op-amps, simply by using a circuit node which is normally connected to analog ground as an additional voltage input, without using additional transistors or stages. However, conventional CMOS CFOA design is still facing some challenges, such as fixed bandwidth, input offset voltage, noise, power consumption and circuit complexity [3-11].

The CFOAs are classified based on four terminal theory instead of conventional op-amps in two port theory [12-13] and so also it could be divided into more types unlike the classification based on two port theory [14], out of which the one of interest is CF-OTA with a voltage buffer. The main advantage of the current feedback OTA is that it can be easily designed from the design of current op-amps, simply by using a circuit node which is normally connected to analog ground as an additional voltage input, without using additional transistor stages. Improvising parameters like bandwidth, slew rate, settling time, input offset voltage and input impedance of CF-OTA has drawn wide attention from researchers. Here we report the design of a CFOA with better performance in terms of gain, bandwidth, slew rate and settling time in $180 \mathrm{~nm}$ and $90 \mathrm{~nm}$. Also a comparison of performance is made between amplifiers with class $\mathrm{AB}$ output stage and source follower output stage.

The rest of the paper is organized as follows: Section 2 illustrates the circuit description and CMOS realization of the proposed CFOA. Section 3 discusses simulation results in two configuration with $90 \mathrm{~nm}$ and $180 \mathrm{~nm}$ technology. Finally concluding points are described in section 4 .

\section{METHODOLOGY}

In four terminal theory, two input and output terminals of opamp are not seen as one port, but it can be seen as four independent terminals that can have different impedance levels. CFOA is a hybrid input current output op-amp and the main advantage of designing CFOA is that it can be easily converted from current op-amps. The current op-amp has normally input stage with only one input and a current output stage. The current input stage of current op-amp is only a current buffer that transfers the input current to the high-impedance node. Converting current input stage into a hybrid input stage implies providing an additional voltage input through which the voltage at the current input can be set. The current output stage converts the voltage at high impedance node into two balanced currents.

\subsection{PROPOSED CMOS CFOA}

In this paper we present a CMOS Current Feedback Operational Amplifier (CFOA) with better bandwidth. In this approach folded cascode op-amp is used as input stage which is shown in Fig. 1 and a preceding voltage buffer is implemented by using class $\mathrm{AB}$ buffer as shown in Fig.2(b). This output stage circuit helps to improve the current drive capability. The use of class $\mathrm{AB}$ buffer helps to decrease the output impedance 
drastically. Also in this paper an observation is done by replacing the output stage with source follower circuit as in Fig.2(a) and it is found that only the slew rate is improved. The design parameters such as bias currents and the gate dimensions are given in Table.1.

The design of input stage starts by designing the folded cascode OTA using $\mathrm{gm} / \mathrm{Id}$ technique in strong inversion region [15-16].

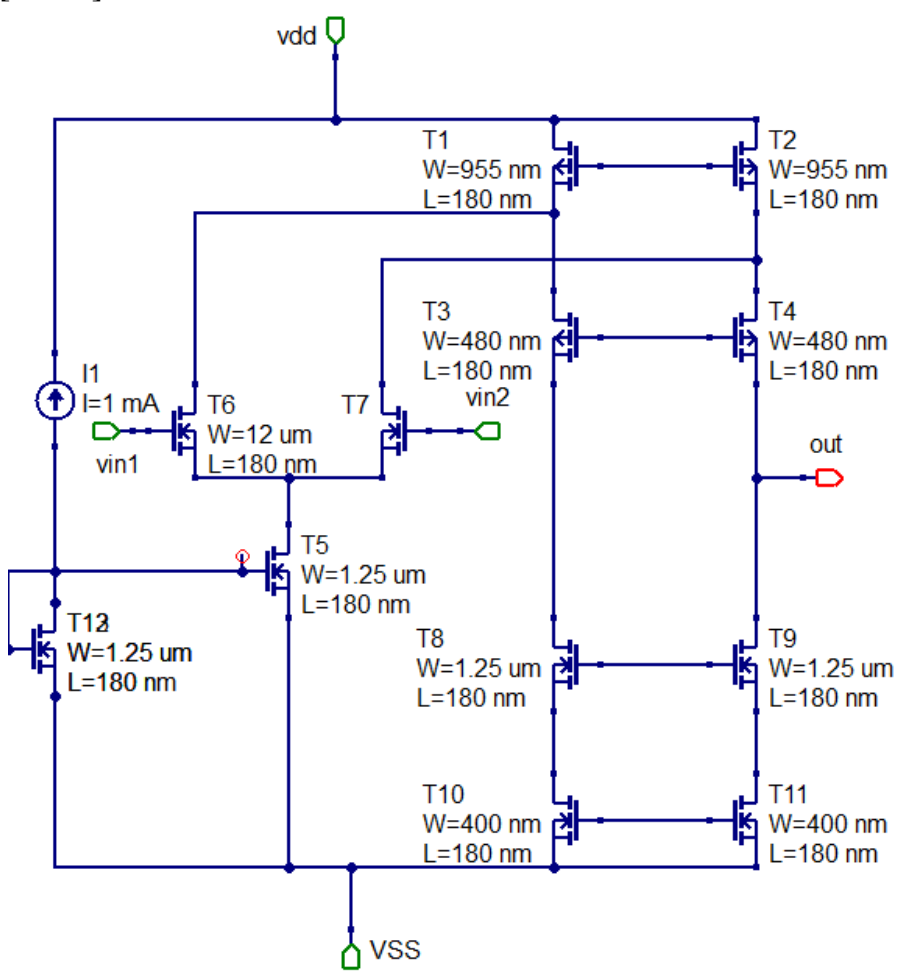

Fig.1. Schematic of CMOS CFOA input stage

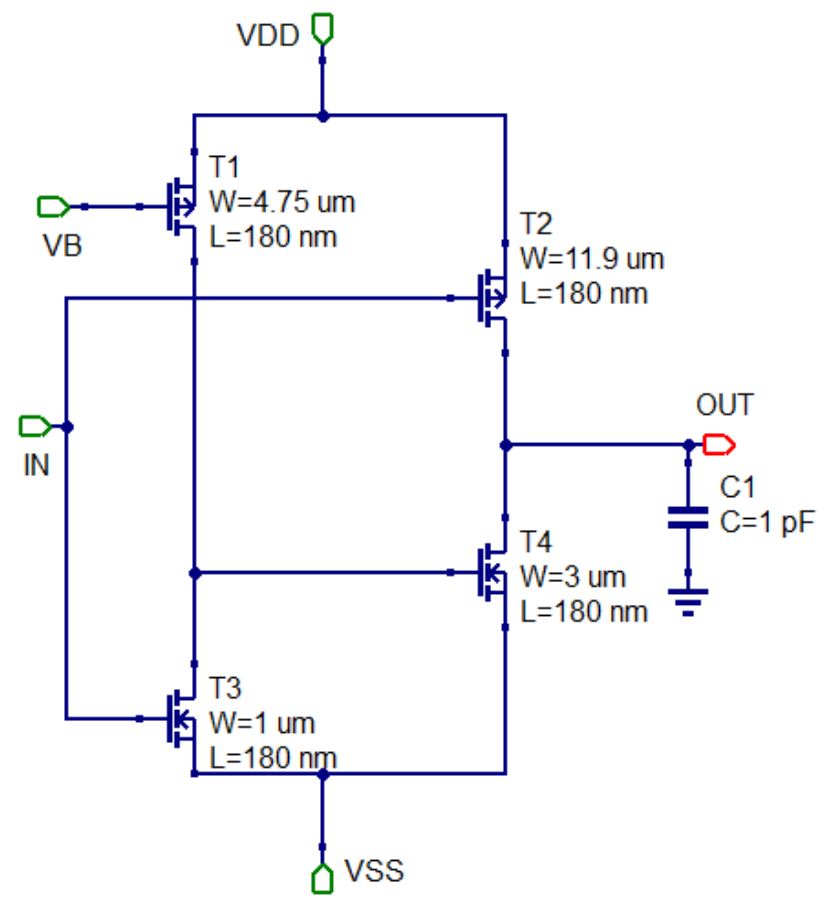

(a) Source follower output

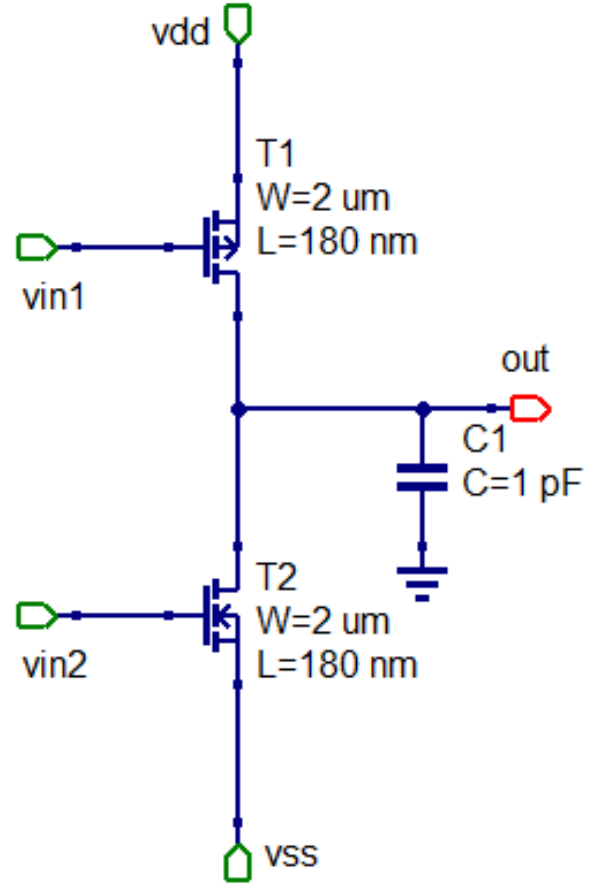

(b) Class AB output buffer

Fig.2. Schematic of CMOS CFOA output stages

In the $G_{m} / I_{d}$ technique, the bias current strongly depends on transconductance of the OTA by the Eq.(1)

$$
g_{m}=\frac{\left\{V_{i n}(+)-V_{i n}(-)\right\}}{I_{d}}
$$

The folded cascod op-amps helps to achieve higher DC gain at the initial stage. The $G B W$ of OTA is given by the Eq.(2)

$$
G B W=\frac{g_{m}}{C_{l}}
$$

The gate dimensions of the transistors $M_{1}$ and $M_{2}$ are related to the $G B W$ of the OTA and it is given by the Eq.(3)

$$
\left(\frac{W}{L}\right)_{1}=\left(\frac{W}{L}\right)_{2}=\frac{g_{m 1}^{2}}{\mu_{n} \operatorname{CoxI}_{d}}=\frac{G B W^{2} C_{l}^{2}}{\mu_{n} \operatorname{CoxI}_{d}}
$$

where, $g_{m}$ is the transconductance of the differential pair transistor, $C_{l}$ is the load capacitance and $\mu_{n}$ Cox is the parameter which depends on the material constant.The current Id is related to the slew rate and load capacitance by the Eq.(4)

$$
I_{d}=S R \times C_{l}
$$

The bias currents in cascode is choosen such that 1.2 to 1.5 times greater than the $I_{d}$ to avoid zero current in cascodes. The gate dimensions of the transistors $M_{4}$ to $M_{7}$ are related to the maximum output voltage of the OTA by the Eq.(5) and Eq.(6)

$$
\begin{aligned}
& \left(\frac{W}{L}\right)_{4}=\left(\frac{W}{L}\right)_{5}=\frac{2 I_{5}}{\mu_{p} \operatorname{Cox} V_{D S 5}^{2}} \\
& \left(\frac{W}{L}\right)_{6}=\left(\frac{W}{L}\right)_{7}=\frac{2 I_{7}}{\mu_{p} \operatorname{Cox} V_{D S 7}^{2}}
\end{aligned}
$$

The gate dimensions of the transistors $M_{8}$ to $M_{11}$ are related to the minimum output voltage of the OTA by the Eq.(7) and Eq.(8) 


$$
\begin{aligned}
& \left(\frac{W}{L}\right)_{8}=\left(\frac{W}{L}\right)_{9}=\frac{2 I_{9}}{\mu_{p} \operatorname{Cox} V_{D S 9}^{2}} \\
& \left(\frac{W}{L}\right)_{10}=\left(\frac{W}{L}\right)_{11}=\frac{2 I_{11}}{\mu_{p} \operatorname{Cox} V_{D S 11}^{2}}
\end{aligned}
$$

The minimum and maximum input common mode is set by Eq.(9) and Eq.(10)

$$
\begin{gathered}
\left(\frac{W}{L}\right)_{3}=\frac{2 I_{3}}{\mu_{n} \operatorname{Cox}\left(V_{i n_{\min }}-V_{s s}-\sqrt{\left(\frac{I_{3}}{\mu_{n} \operatorname{Cox}}\right)}-V_{t}\right)^{2}} \\
\left(\frac{W}{L}\right)_{4}=\frac{2 I_{3}}{\mu_{p} \operatorname{Cox}\left(V_{d d}-V_{i n_{\max }}+V_{t}\right)^{2}}
\end{gathered}
$$

Table.1. Gate dimensions and biasing currents in proposed CMOS CFOA

\begin{tabular}{|c|c|c|c|c|}
\hline \multirow{2}{*}{ Transistors } & \multicolumn{3}{|c|}{ Gate dimensions and biasing currents } \\
\cline { 2 - 5 } & \multicolumn{2}{|c|}{$L=\mathbf{1 8 0 n m}$} & \multicolumn{2}{c|}{$L=\mathbf{9 0 n m}$} \\
\cline { 2 - 5 } & Width & Current $(\mu \mathbf{A})$ & Width & Current $(\mu \mathbf{A})$ \\
\hline$M_{1}, M_{2}$ & $12 \mu \mathrm{m}$ & 50 & $30 \mu \mathrm{m}$ & 25 \\
\hline$M_{3}$ & $1.25 \mu \mathrm{m}$ & 100 & $500 \mathrm{~nm}$ & 50 \\
\hline$M_{12}$ & $1.25 \mu \mathrm{m}$ & 100 & $690 \mathrm{~nm}$ & 50 \\
\hline$M_{4}, M_{5}$ & $955 \mathrm{~nm}$ & 100 & $445 \mathrm{~nm}$ & 50 \\
\hline$M_{6}, M_{7}$ & $480 \mathrm{~nm}$ & 50 & $300 \mathrm{~nm}$ & 25 \\
\hline$M_{8}, M_{9}$ & $1.25 \mu \mathrm{m}$ & 50 & $1.75 \mu \mathrm{m}$ & 25 \\
\hline$M_{10}, M_{11}$ & $400 \mathrm{~nm}$ & 50 & $1.75 \mu \mathrm{m}$ & 25 \\
\hline
\end{tabular}

\section{RESULTS AND DISCUSSIONS}

CFOA having high bandwidth with very low input offset voltage, low settling time, higher unity gain bandwidth product, $-3 \mathrm{~dB}$ bandwidth and improved slew rate is proposed in this paper. Simulation was carried out in 3 different ways.

- CFOA with class AB output buffer stage in $180 \mathrm{~nm}$ technology

- CFOA with source follower output buffer stage in $180 \mathrm{~nm}$ technology

- CFOA with class AB output buffer stage in 90nm technology $(50 \mu \mathrm{A})$

From the simulation of CFOAs in 180nm CMOS process, excellent frequency responses in hundreds of $\mathrm{MHz}$ are obtained. The simulation of CFOA with class AB buffer gives DC gain of $61 \mathrm{~dB}$ with a unity gain bandwidth of $714 \mathrm{MHz}$ is shown in Fig.3. The simulation of CFOA with source follower gives DC gain of $59.48 \mathrm{~dB}$ with a unity gain bandwidth of $626 \mathrm{MHz}$ as shown in Fig.4. The existing feature of the CFOA with class AB buffer is that one can change the gain independent of the bandwidth (or shows less dependency).

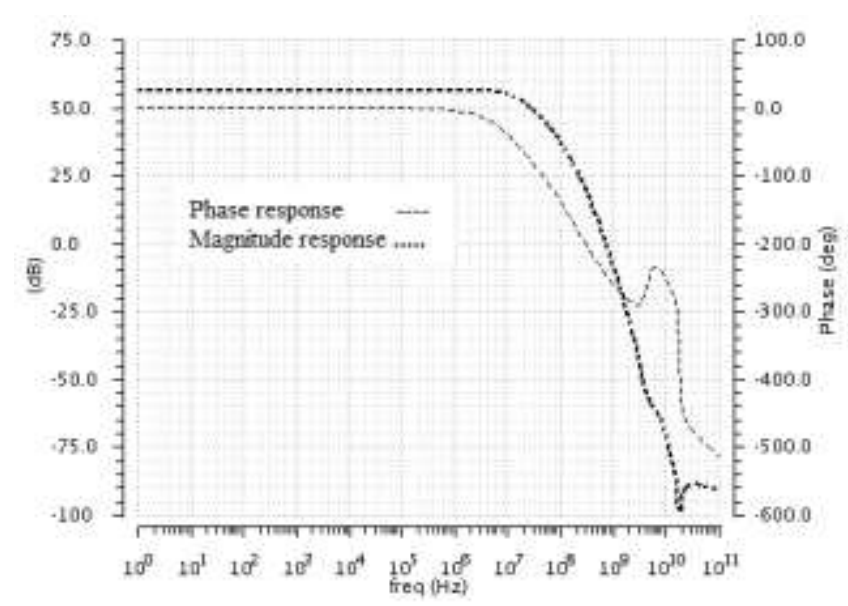

Fig.3. Open-loop frequency response of the proposed CMOS CFOA with class $A B$ buffer

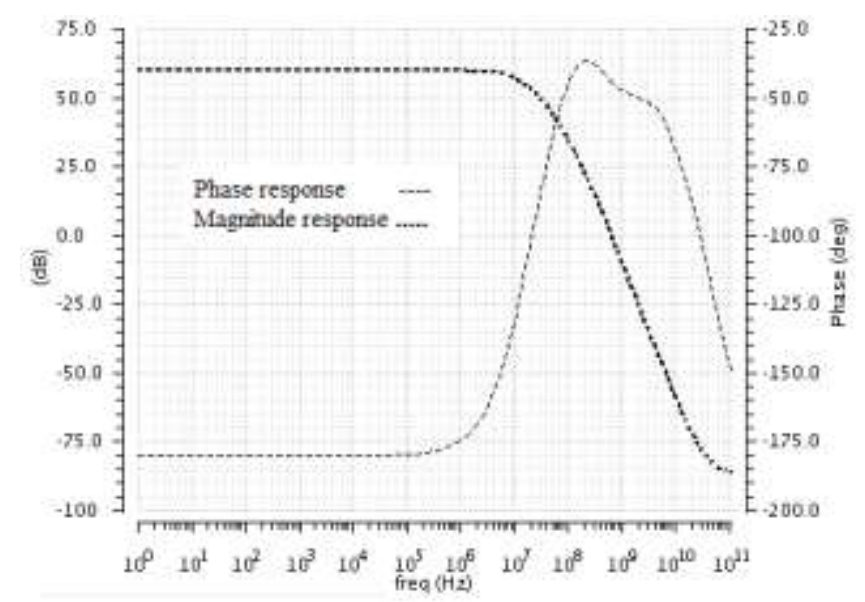

Fig.4. Open-loop frequency response of the proposed CMOS CFOA with source follower

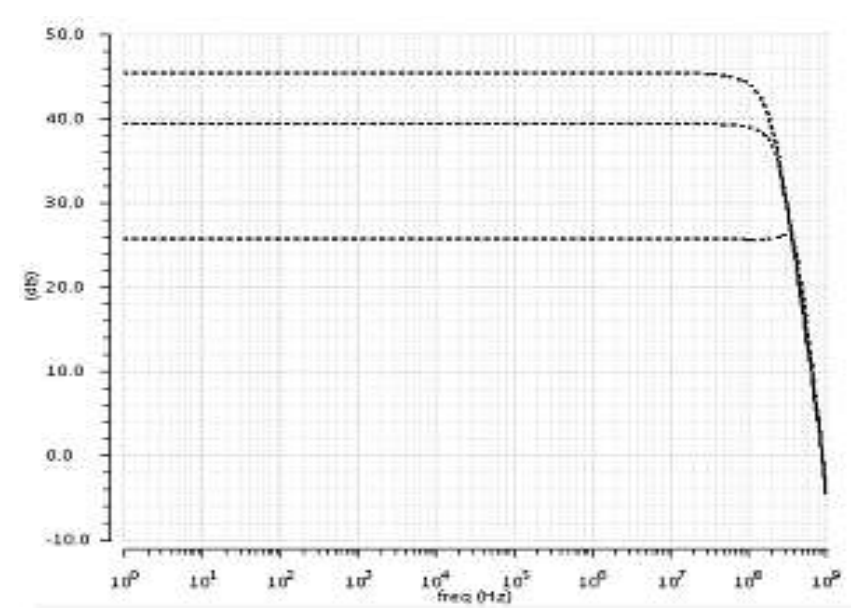

Fig.5. Closed-loop frequency response of the CFOA with class $\mathrm{AB}$ buffer for different gains 


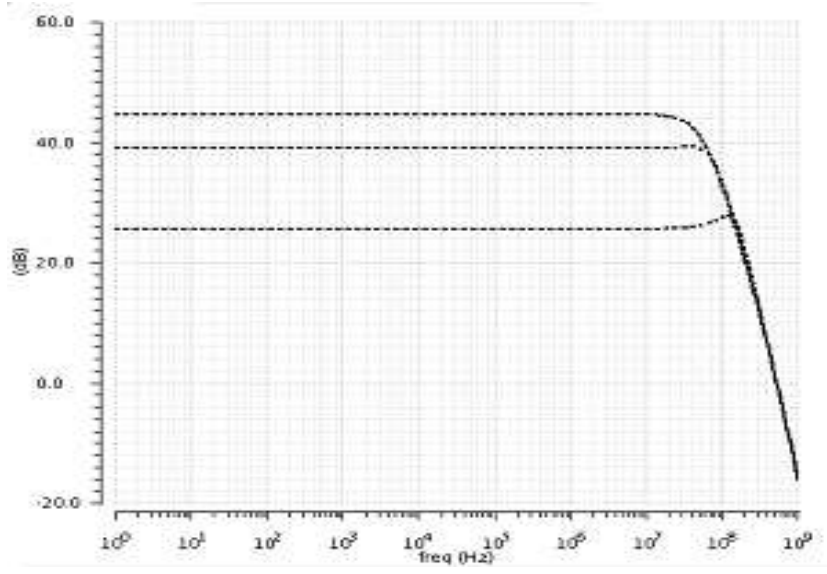

Fig.6. Closed-loop frequency response of the proposed CMOS CFOA with source follower for different gains

Table.2. Comparison between different configurations

\begin{tabular}{|c|c|c|c|}
\hline \multirow{2}{*}{ Parameters } & \multicolumn{2}{|c|}{$\mathbf{1 8 0 n m}$} & 90nm \\
\cline { 2 - 4 } & $\begin{array}{c}\text { CFOA } \\
\text { (Class AB) }\end{array}$ & $\begin{array}{c}\text { CFOA (Source } \\
\text { follower) }\end{array}$ & $\begin{array}{c}\text { CFOA } \\
\text { (Class AB) }\end{array}$ \\
\hline Supply V & $2.5 \mathrm{~V}$ & $2.5 \mathrm{~V}$ & $1.2 \mathrm{~V}$ \\
\hline I bias & $100 \mu \mathrm{A}$ & $100 \mu \mathrm{A}$ & $50 \mu \mathrm{A}$ \\
\hline Bandwidth $(\mathrm{MHz})$ & 80.97 & 59.48 & 230 \\
\hline GBW in MHz & 714 & 626 & 910 \\
\hline Settling time & $40 \mathrm{~ns}$ & $80 \mathrm{~ns}$ & $70 \mathrm{~ns}$ \\
\hline Slew rate & $30 \mathrm{~V} / \mu \mathrm{s}$ & $110 \mathrm{~V} / \mu \mathrm{s}$ & $90 \mathrm{~V} / \mu \mathrm{s}$ \\
\hline Input V Range & -1 to 1 & -1 to 1 & -.8 to .9 \\
\hline Technology & $180 \mathrm{~nm}$ & $180 \mathrm{~nm}$ & $90 \mathrm{~nm}$ \\
\hline
\end{tabular}

By simulating the circuit with class $\mathrm{AB}$ buffer as the output stage, an improved closed loop response is obtained as in Fig.5. Then observed the simulated closed loop response obtained by replacing class $\mathrm{AB}$ buffer output stage with source follower as in Fig.6. From the simulation of CFOAs in 90nm CMOS process, a further improved frequency response is obtained. The DC gain of $32.13 \mathrm{~dB}$ and unity gain bandwidth of $910 \mathrm{MHz}$ is obtained as shown in Fig.7. The closed loop response of the same for various gain and $3 \mathrm{~dB}$ bandwidth is also given in Fig.8.

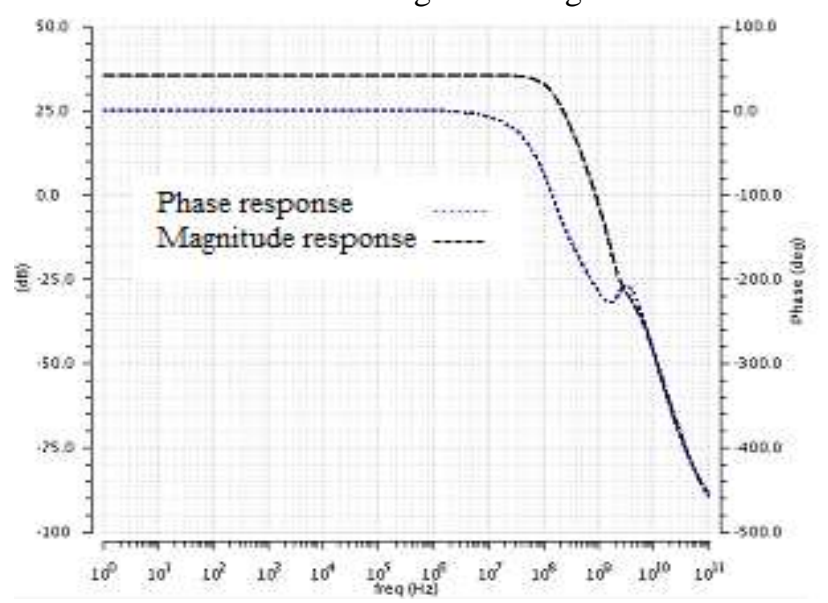

Fig.7. Open-loop frequency response of the proposed CMOS CFOA in $90 \mathrm{~nm}$ process

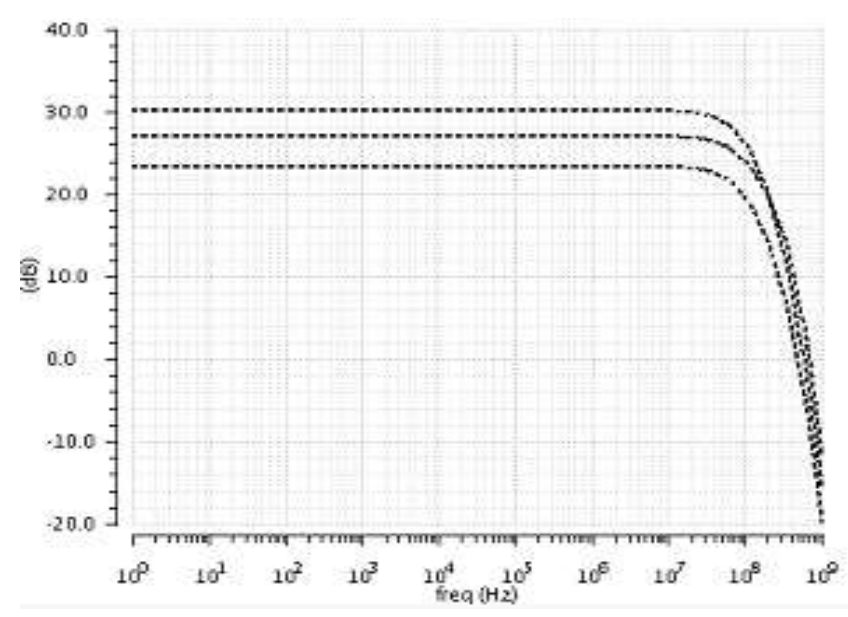

Fig.8. Closed-loop frequency response of the proposed CMOS CFOA with different gains

\section{CONCLUSION}

A CMOS CFOA having a better bandwidth is obtained and the design of proposed CFOA is carried out in both $90 \mathrm{~nm}$ and $180 \mathrm{~nm}$ CMOS process, and the output stages are implemented in Class $\mathrm{AB}$ cross coupled amplifier and source follower. The simulation results show improved gain, bandwidth, slew rate and settling time. It is obvious from the results that the CFOA with class AB output stage provides better frequency response and it can be effectively used in active filter design or high speed data converters.

\section{REFERENCES}

[1] A.H. Madian, S.A. Mahmoud and A.M. Soliman, "Configurable Analog Block Based on CFOA and Its Application", WSEAS Transactions on Electronics, Vol. 5, No. 6, pp. 220-225, 2008.

[2] Byungjoon Park, Sangsu Jin, Daechul Jeong, Jooseung Kim, Yunsung Cho, Kyunghoon Moon and Bumman Kim, "Highly Linear mm-Wave CMOS Power Amplifier", IEEE Transactions on Microwave Theory and Techniques, Vol. 64, No. 12, pp. 4535-4544, 2016.

[3] H.L. Chao and D.S. Ma, "CMOS Variable-Gain WideBandwidth CMFB-Free Differential Current Feedback Amplifier for Ultrasound Diagnostic Applications", Proceedings of IEEE International Symposium on Circuits and Systems, pp. 649-652, 2006.

[4] G. Giustolisi, G. Palmisano, G. Palumbo and S. Pennisi, "High-Drive CMOS Current-Feedback Opamp", Proceedings of $40^{\text {th }}$ Midwest Symposium on Circuits and Systems, pp. 229-232, 1997.

[5] G. Palumbo and S. Pennisi, "Current-Feedback Amplifiers versus Voltage Operational Amplifiers", IEEE Transactions on Circuits and Systems, Vol. 48, No. 5, pp. 617-623, 2001.

[6] S. Pennisi, "High-Performance CMOS Current Feedback Operational Amplifier", Proceedings of IEEE International Symposium on Circuits and Systems, Vol. 2, pp. 1573-1576, 2005.

[7] J. Mahattanakul and C. Toumazou, "A Theoretical Study of the Stability of High Frequency Current Feedback Op-Amp 
Integrators", IEEE Transactions on Circuits and Systems, Vol. 43, No. 1, pp. 1-12, 1996.

[8] M. Djebbi, A. Assi and M. Sawan, "An Offset-Compensated Wide-Bandwidth CMOS Current-Feedback Operational Amplifier", Proceedings of Canadian Conference on Electrical and Computer Engineering, pp. 73-76, 2003.

[9] J. Zhu, M. Sawan and K. Arabi, "An Offset Compensated CMOS Current-Feedback Operational Amplifier", Proceedings of IEEE International Symposium on Circuits and Systems, pp. 1552-1555, 1995.

[10] A.M. Ismail and A.M. Soliman, "Novel CMOS Current Feedback Op-Amp Realization Suitable for High Frequency Applications", IEEE Transactions on Circuits and Systems I: Fundamental Theory and Applications, Vol. 47, No. 6, pp. 918-921, 2000.

[11] S. Selvanayagam and F.J. Lidgey, "Wide Bandwidth CMOS Current Feedback Op-Amp for Inverting Applications", IEEE Electronics Letters Journal, pp. 1-4, 1996.
[12] Alison Payne and Chris Toumazou, "Analog Amplifiers: Classification and Generalization", IEEE Transactions on Circuits and Systems I: Fundamental Theory and Applications, Vol. 43, No. 1, pp. 43-50, 1996.

[13] Rafael Cabeza and Alfonso Carlosena, "Analog Universal Active Device: Theory, Design and Applications", Analog Integrated Circuits and Signal Processing, Vol. 12, No. 2, pp. 153-168, 1997.

[14] H. Schmid, "The Current-Feedback OTA", Proceedings of IEEE International Symposium on Circuits and Systems, pp. 655-658, 2001.

[15] P.E. Allen and D.R. Holberg, "CMOS Analog Circuit Design", $2^{\text {nd }}$ Edition, Oxford University Press, 2004.

[16] T. Singh, M. Kaur and G. Singh, "Design and Analysis of CMOS Folded-Cascode OTA using $\mathrm{G}_{\mathrm{m}} / \mathrm{I}_{\mathrm{d}}$ Technique", International Journal of Electronics and Computer Science Engineering, Vol. 1, No. 2, pp. 727-733, 2012. 